Supporting Information

\title{
General Method for Post-Synthetic Modification of Oligonucleotides Based on
}

Oxidative Amination of 4-Thio-2'-deoxyuridine

Jingyi Wang, Jiachen Shang, Yu Xiang and Aijun Tong*

Department of Chemistry, Beijing Key Laboratory for Microanalytical Methods and Instrumentation, Key Laboratory of Bioorganic Phosphorus Chemistry and Chemical Biology (Ministry of Education), Tsinghua University, Beijing 100084, China

Email: tongaj@mail.tsinghua.edu.cn 


\section{Materials and Instruments}

\section{Chemicals}

All chemicals were used as received without further purification unless specified. Ethylenediamine tetraacetic acid disodium salt (EDTA), sodium dihydrogen phosphate dihydrate $\left(\mathrm{NaH}_{2} \mathrm{PO}_{4} \cdot 2 \mathrm{H}_{2} \mathrm{O}\right)$, disodium hydrogen phosphate dodecahydrate $\left(\mathrm{Na}_{2} \mathrm{HPO}_{4} \cdot 12 \mathrm{H}_{2} \mathrm{O}\right)$, sodium chloride $(\mathrm{NaCl})$, sodium hydroxide $(\mathrm{NaOH})$, concentrated hydrochloric acid $(\mathrm{HCl})$, anhydrous sodium sulfate $\left(\mathrm{Na}_{2} \mathrm{SO}_{4}\right)$, ethylenediamine and anhydrous copper sulfate $\left(\mathrm{CuSO}_{4}\right)$ were purchased from Beijing Chemical Works (Beijing, China). Dichloromethane (DCM), ethyl acetate (EtOAc) and acetone were purchased from Greagent (Shanghai, China). Dimethylformamide (DMF), urea and tri(hydroxymethyl)aminomethane (Tris) were purchased from Amresco (Solon, USA). Trifluoroethylamine (TFEA) was purchased from Shaoyuan (Shanghai, China). Sodium periodate $\left(\mathrm{NaIO}_{4}\right), 11$-azido-3,6,9-trioxaundecan-1-amine (Az-PEG3-NH 2$)$ and chromatographic grade glacial acetic acid (HAc) were purchased from Aladdin (Shanghai, China). 2-[2(propargyloxy)ethoxy]ethylamine (Alk-PEG2-NH2) was purchased from Ark (Chicago, USA). Aniline (Ph$\mathrm{NH}_{2}$ ) was purchased from Adamas-beta (Shanghai, China). Sodium ascorbate and 1-aminopyrene (Pyr$\mathrm{NH}_{2}$ ) were purchased from Alfa Aesar (Shanghai, China). Dansyl chloride (Dan-Cl) and 5-aminofluorescein (Fl-NH${ }_{2}$ ) were purchased from Heowns (Tianjin, China). Benzylamine (Bz-NH2) was purchased from J\&K (Beijing, China). Chlroform- $d\left(\mathrm{CDCl}_{3}\right)$ and dimethyl sulfoxide- $d$ (DMSO- $\left.d_{6}\right)$ were purchased from Macklin (Shanghai, China). 4-thiouracil, ALKYNE-FLUOR 488 (Alk488), AZIDE-FLUOR 545 (Az545) and tris(3hydroxypropyltriazolylmethyl)amine (THPTA) were purchased from Sigma-Aldrich (St. Louis, USA). 33\% Ethylamine $\left(\mathrm{Et}_{-} \mathrm{NH}_{2}\right)$ aqueous solution and N-biotinyl-3,6-dioxaoctane-1,8-diamine (Bio-PEG2- $\left.\mathrm{NH}_{2}\right)$ were purchased from TCI (Shanghai, China). 3 M NaAc-HAc buffer (pH 5.2) and 30\% PAGE pre-solution (29:1) were purchased from Solarbio (Beijing, China). 10000×SYBR Gold and 10×TBE were purchased from Biolite (Tianjin, China) and Leagene (Beijing, China) respectively. Chromatographic grade triethylamine (TEA) was purchased from Innochem (Beijing, China). Gradient grade acetonitrile was purchased from Hipure Chem (Elmsford, USA).

\section{Oligonucleotides and other materials}

Oligonucleotides were synthesized and purified by either Sangon Biotech Co., Ltd. (Shanghai, China) or Trilink Biotech Co., Ltd. (San Diego, USA). The sequences used were as follows.

4SdU ON: TCACGCT / \{4-Thio-2'-deoxyuridine $\} /$ TCACCGTGG 
dU ON: TCACGCT/\{deoxyuridine\}/TCACCGTGG

dC ON: TCACGCTCTCACCGTGG

Amicon-3k centrifugal filters were purchased from Millipore (Billerica, USA). Streptavidin magnetic beads was purchased from New England Biolabs (Ipswich, USA).

\section{Instruments}

The reactions of nucleic acids were carried on a ProFlex ${ }^{\mathrm{TM}}$ Base PCR system (Singapore). All absorbance spectra were measured on a JASCO V-550 UV-visible spectrometer (Tokyo, Japan), and all fluorescence spectra were recorded on a JASCO FP-8600 fluorescence spectrometer (Tokyo, Japan). All the gels were imaged on a Bio-Rad ChemiDoc ${ }^{\mathrm{TM}}$ XRS+ system. The NMR spectra was recorded on a JOEL JNM-ECA400 spectrometer (Tokyo, Japan). Mass spectra of nucleic acids was obtained on a SHIMADZU MALDI-TOF mass spectrometer (Kyoto, Japan). HPLC analysis were conducted on a JASCO LC-Net II/ADC series equipped with an Inertsil ODS-SP 250×4.6 column and a UV-2070 UV/Vis detector. 


\section{Additional Figures}

(a)

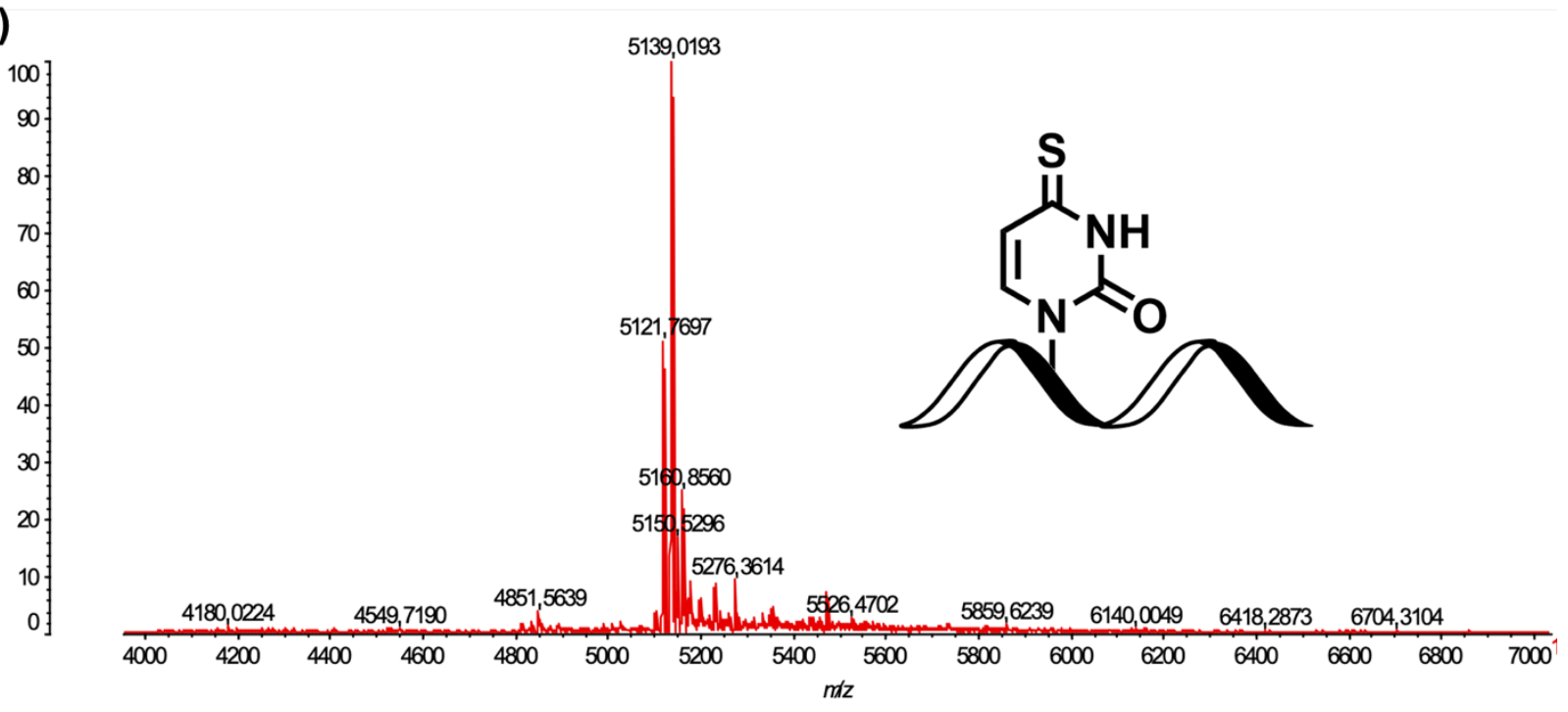

(b)

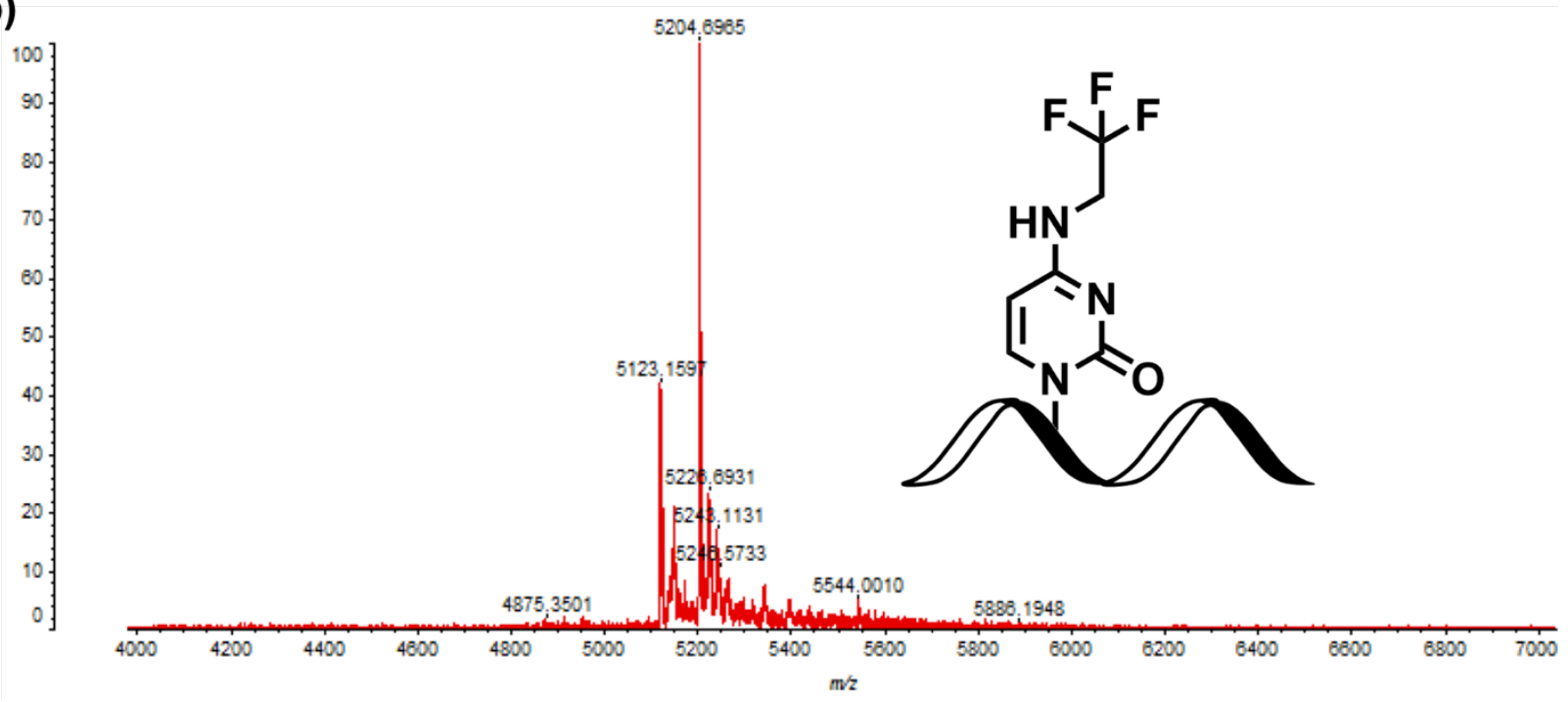

Figure S1. MALDI-TOF MS analysis of 4SdU ON before (a, 4SdU ON, calc. 5139.4, found 5139.0) and after (b, TFE-dC ON, calc. 5204.4, found 5204.7) the oxidative amination treatment. 
(a)
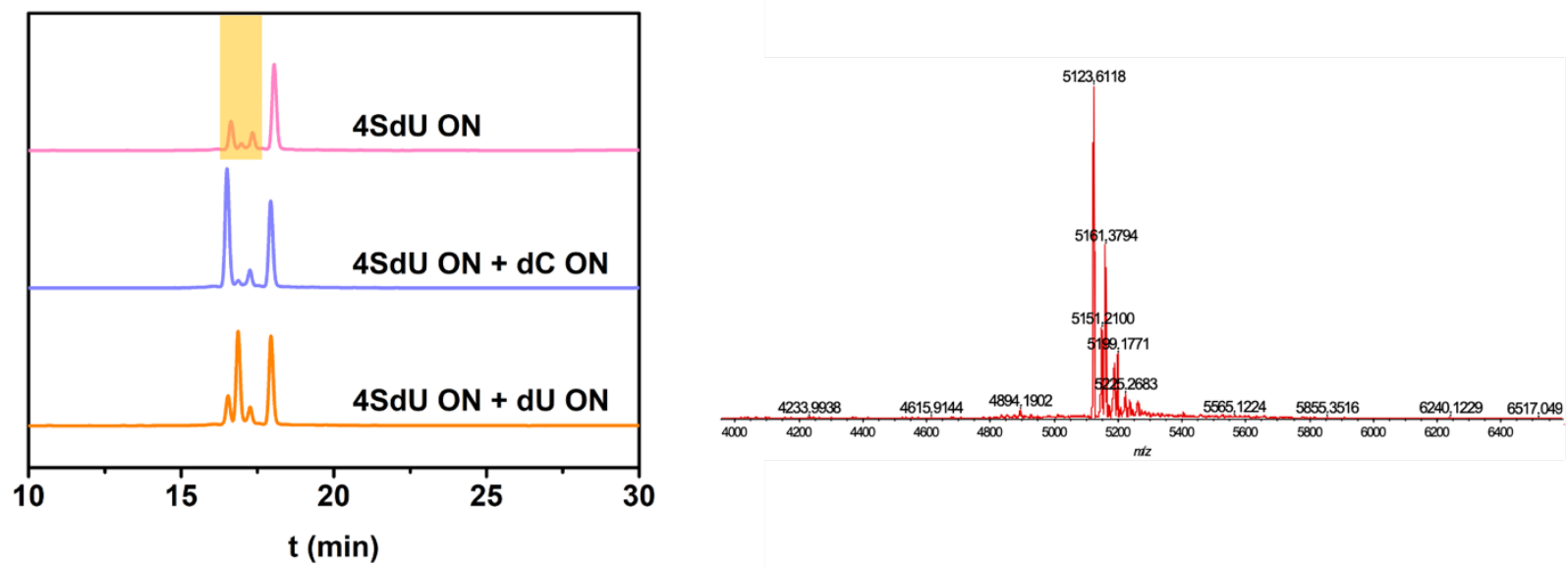

(b)
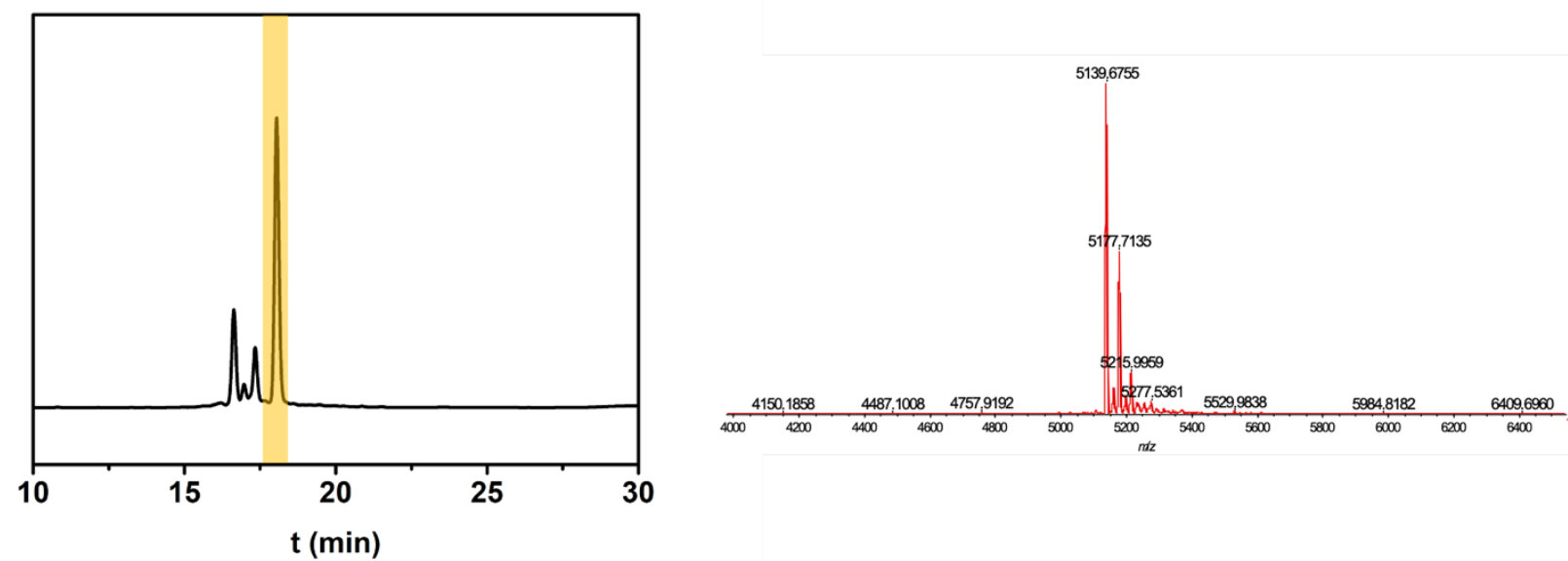

(c)
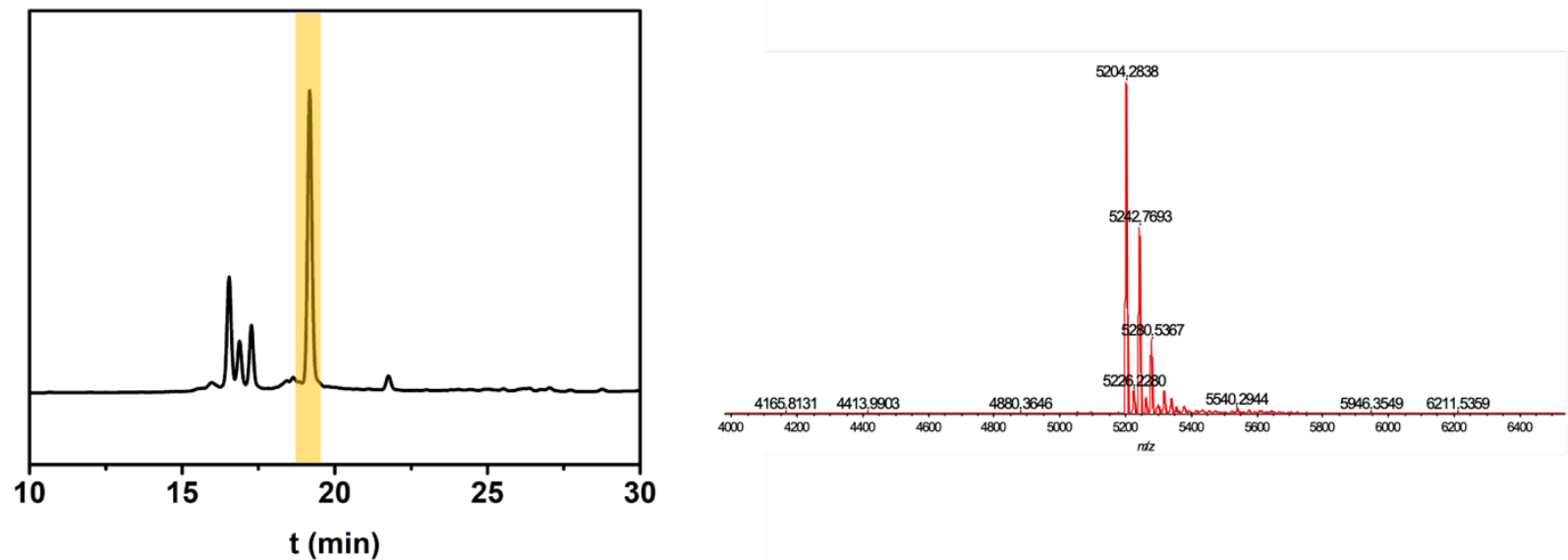

Figure S2. HPLC-UV analysis of impurities (a) and reactant (b) in starting material 4SdU ON as well as product TFE-dC ON (c). The highlight peaks were collected for MALDI-TOF MS analysis. The impurities in the 4SdU ON we used were characterized mainly as dC ON and dU ON via HPLC-UV through standard additions. Their molecular weights were calculated as 5122.4 and 5123.4 respectively, and the observed mass of impurities was 5123.6. The pure reactant 4SdU ON (calc. 5139.4, found 5139.7) and product TFEdC ON (calc. 5204.4, found 5204.3) were isolated by HPLC. Additional peaks in mass spectra ([M] +38 $\mathrm{Da}$ ) were attributed to oligonucleotides replacing the hydrogen with potassium. 
(a)

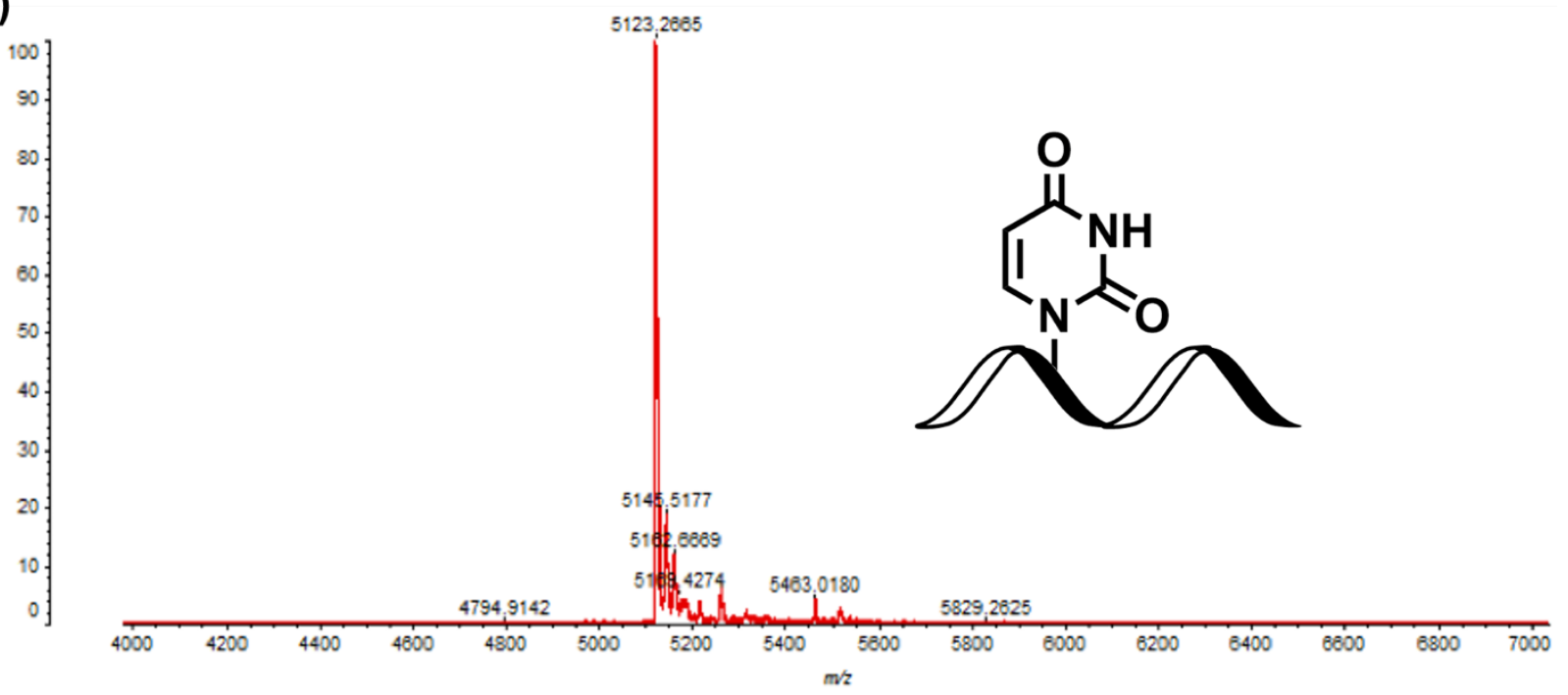

(b)

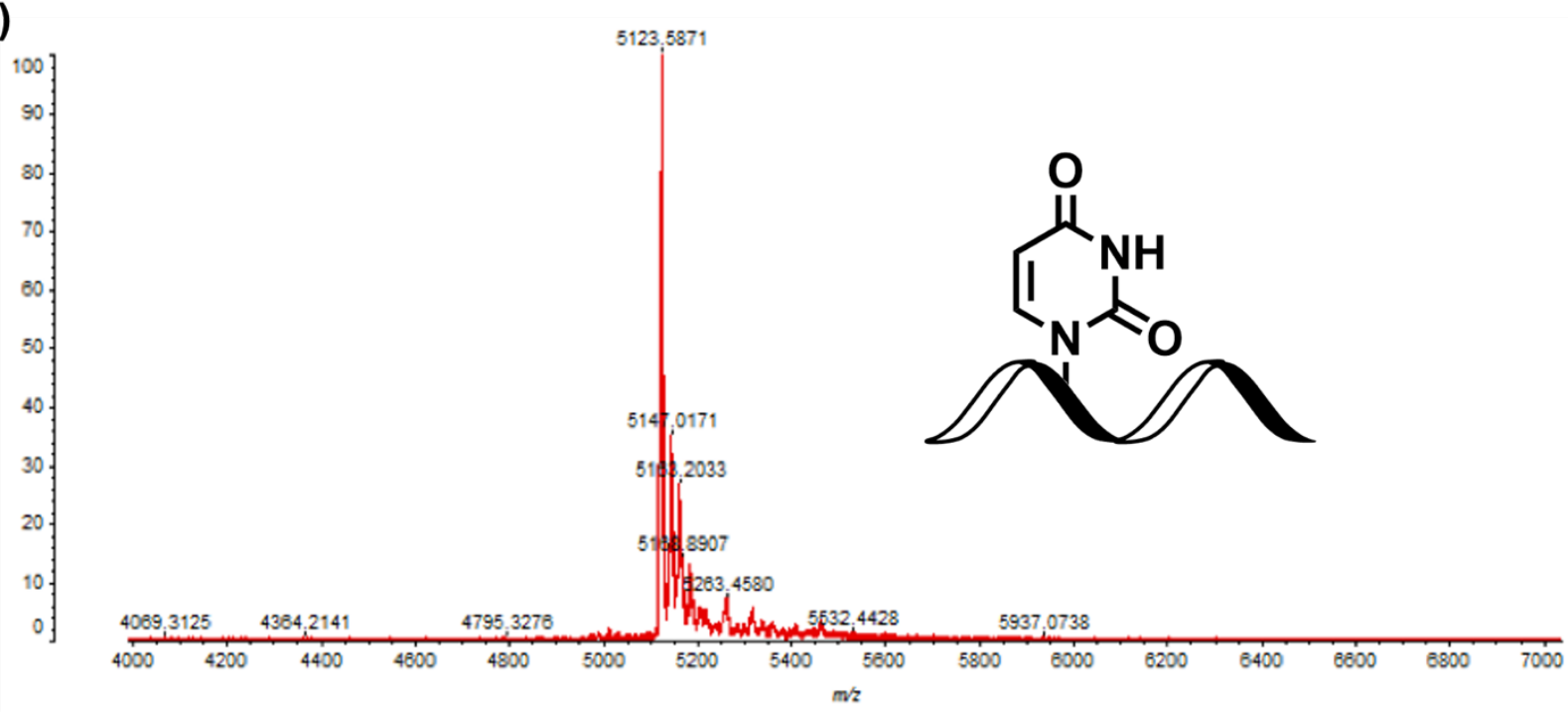

Figure S3. MALDI-TOF MS analysis of dU ON before (a, dU ON, calc. 5123.4, found 5123.3) and after (b, dU ON, calc. 5123.4, found 5123.6) the oxidative amination treatment. 

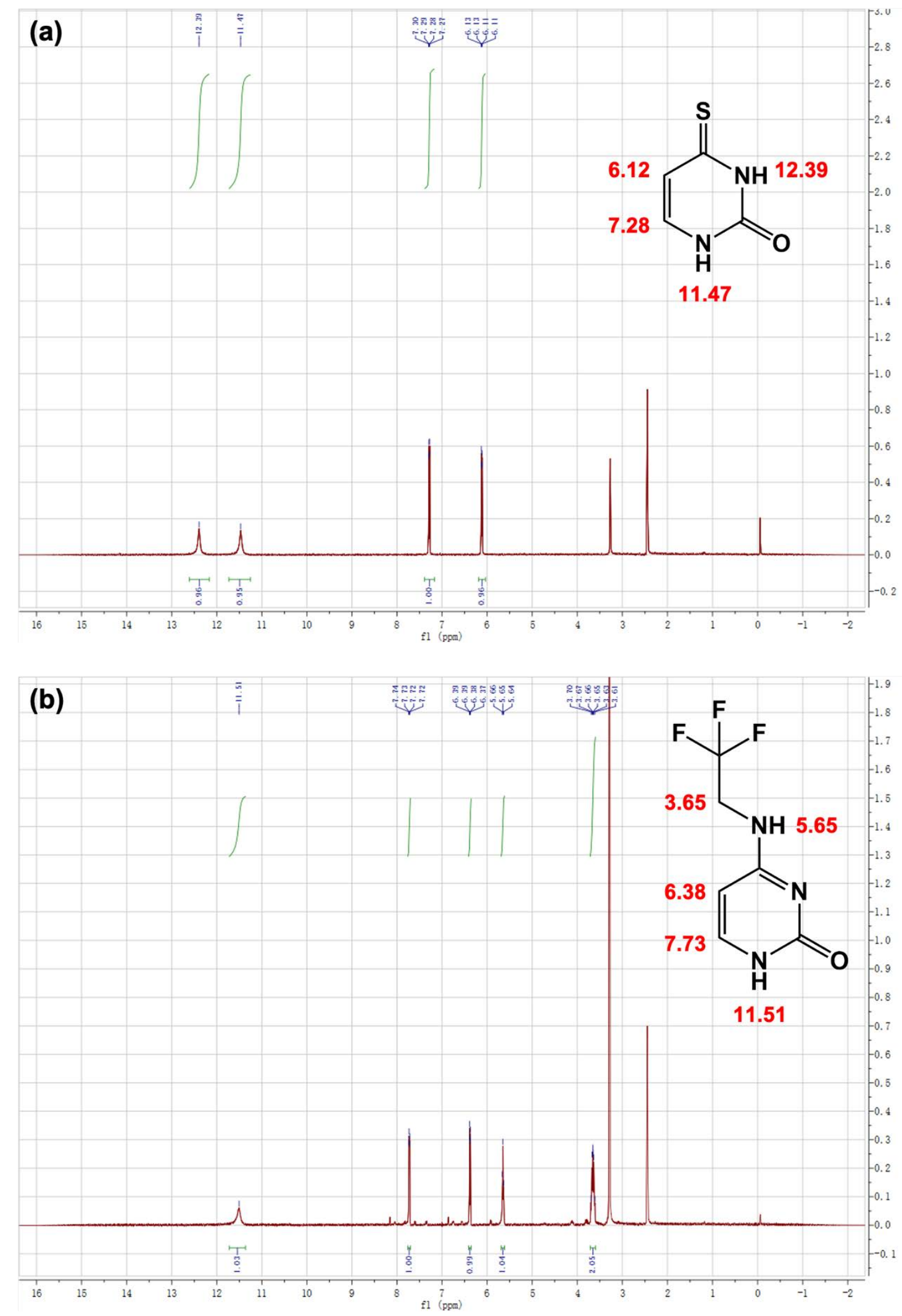

Figure S4. ${ }^{1} \mathrm{H}$ NMR spectrum of 4-thiouracil before (a) and after (b) oxidative amination reaction in $d_{6}$ DMSO. After treated with $\mathrm{NaIO}_{4}$ and TFEA, 4-thiouracil was converted to the TFE-derived cytosine as the structure displayed. The peaks appeared at $2.5 \mathrm{ppm}$ and $3.3 \mathrm{ppm}$ belonged to solvent peak of DMSO and $\mathrm{H}_{2} \mathrm{O}$ respectively. 
(a)

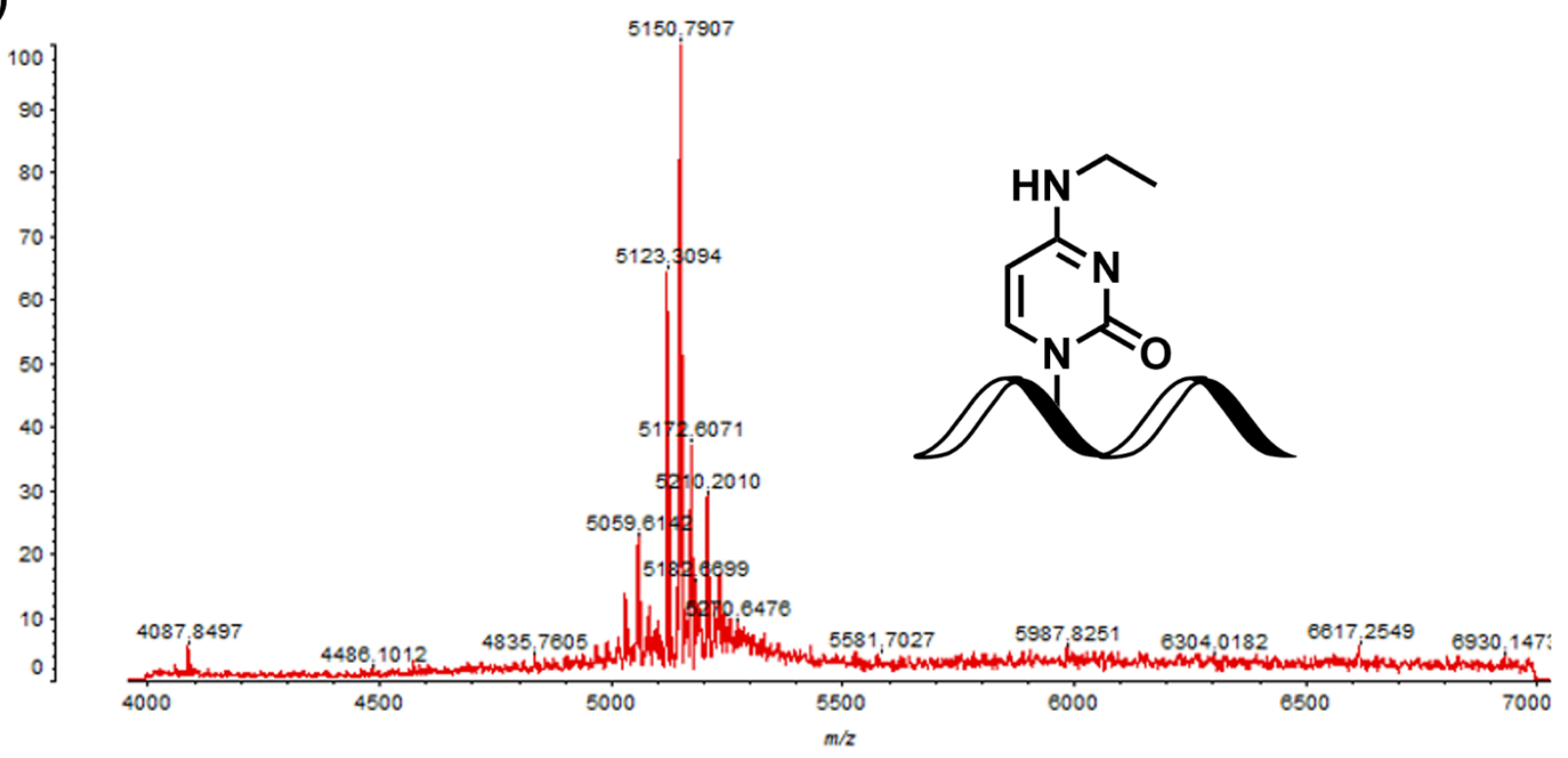

(b)

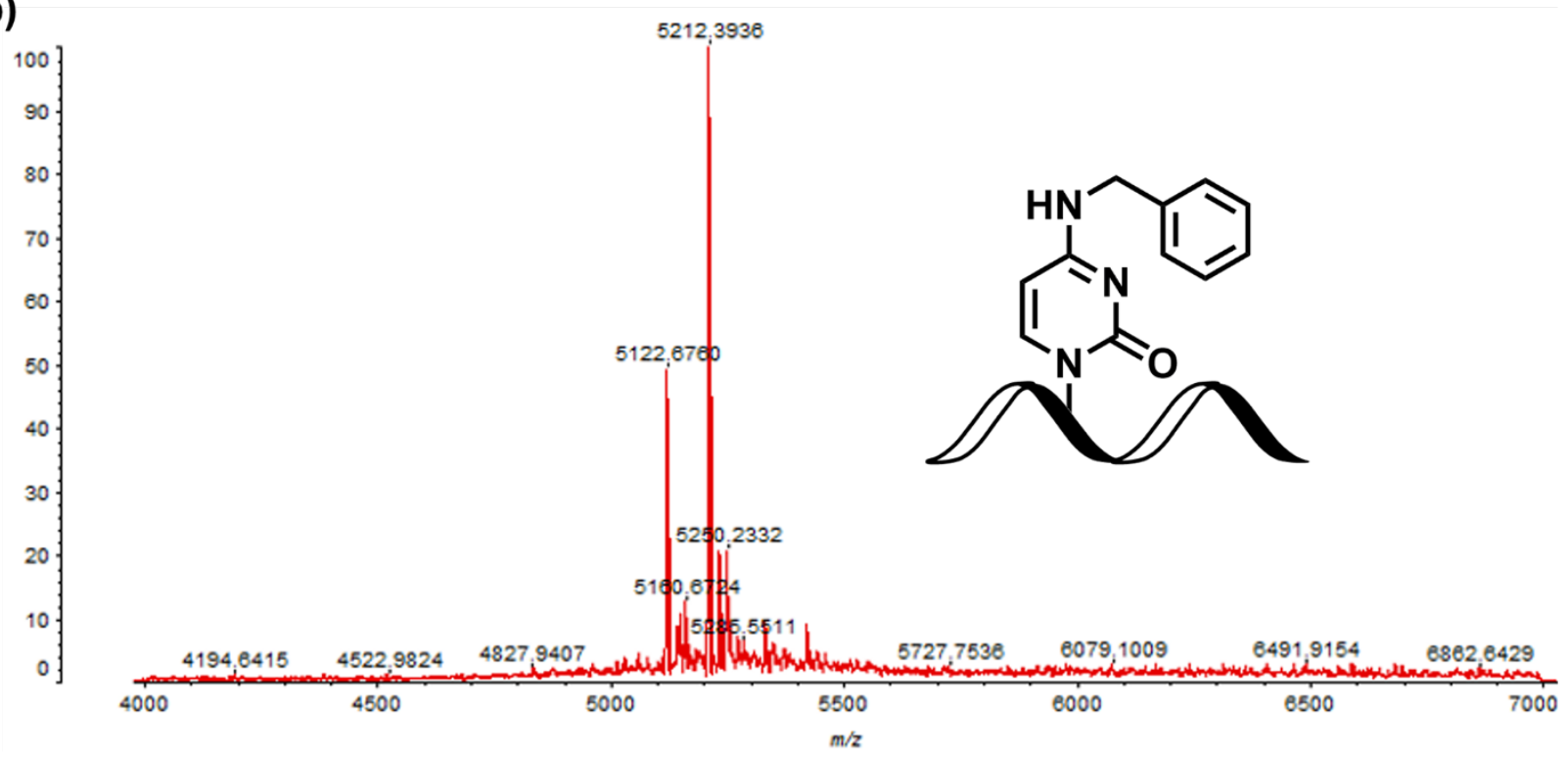

Figure S5. MALDI-TOF MS analysis of 4SdU ON derived with ethylamine $\left(\mathrm{Et}^{\left.-\mathrm{NH}_{2}\right)}\right.$ and benzylamine (Bz-NH ${ }_{2}$ ), namely Et-dC ON (a, calc. 5150.5, found 5150.8) and Bz-dC ON (b, calc. 5212.6, found 5212.4). 
(a)
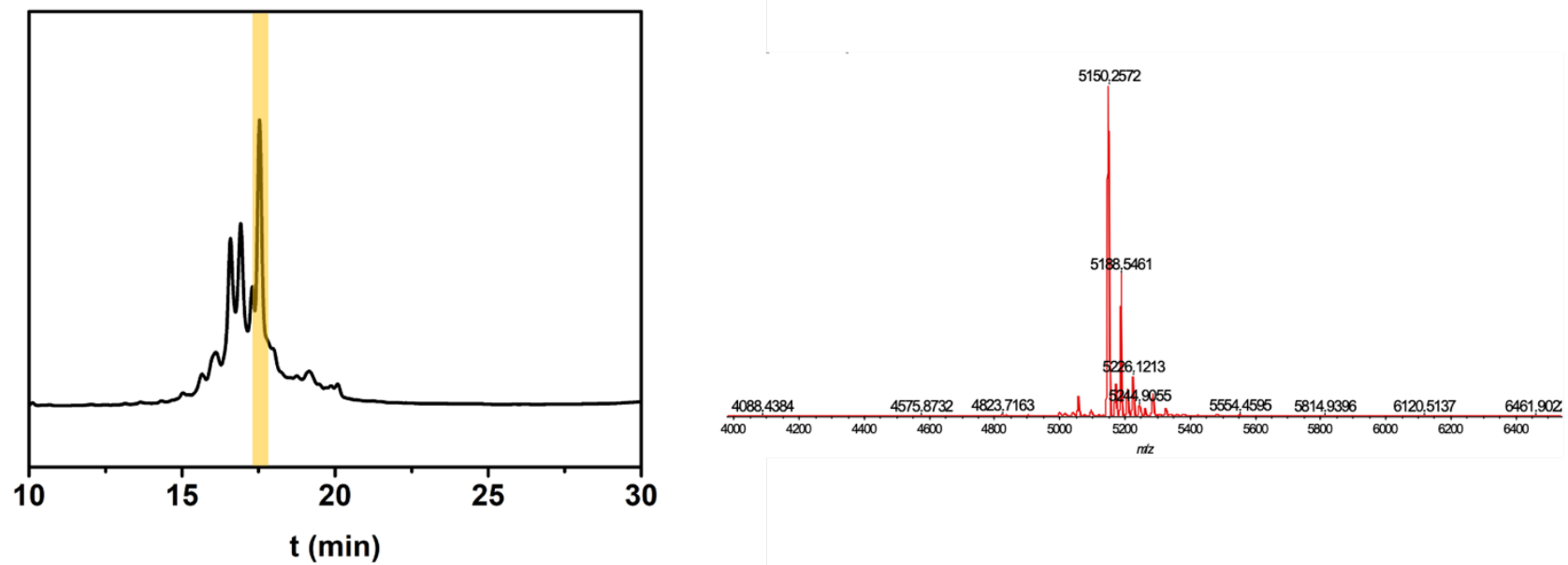

(b)
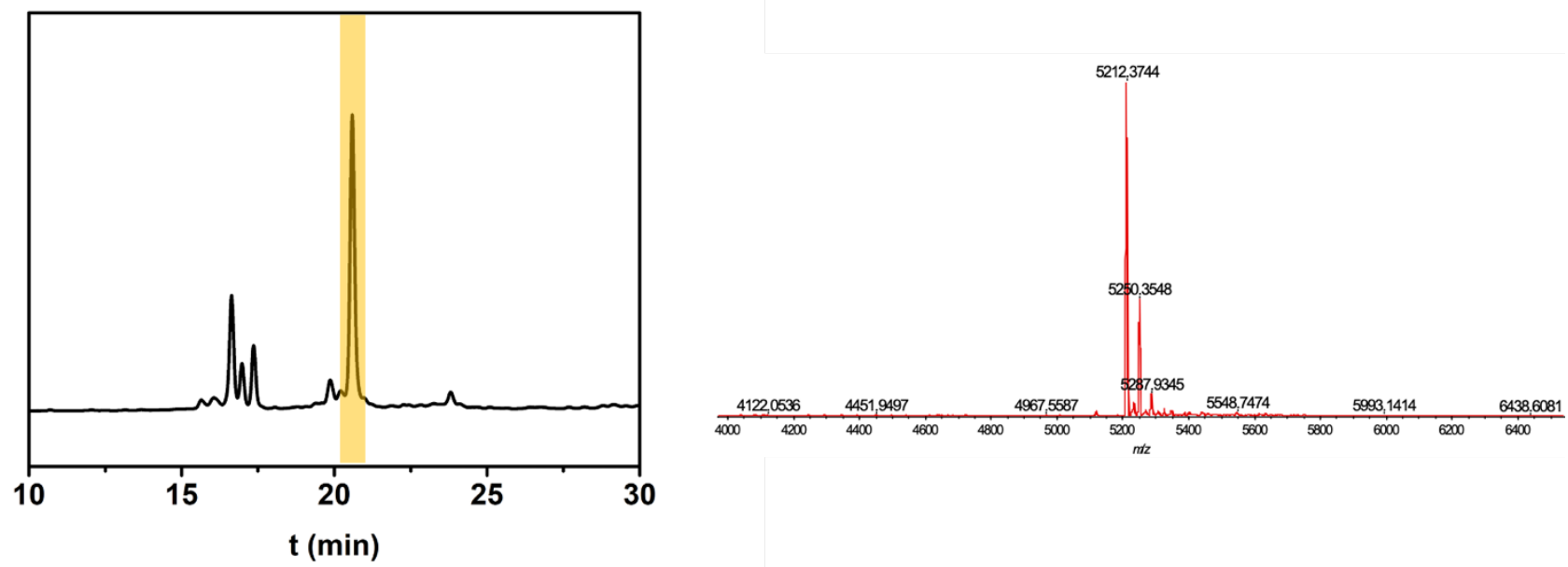

Figure S6. HPLC-UV analysis of Et-dC ON (a) and Bz-dC ON (b). The highlight peaks were collected for MALDI-TOF MS analysis. The pure products Et-dC ON (calc. 5150.5, found 5150.3) and Bz-dC ON (calc. 5212.6, found 5212.4) were isolated by HPLC. Additional peaks in mass spectra ([M] + $38 \mathrm{Da})$ were attributed to oligonucleotides replacing the hydrogen with potassium. 
(a)

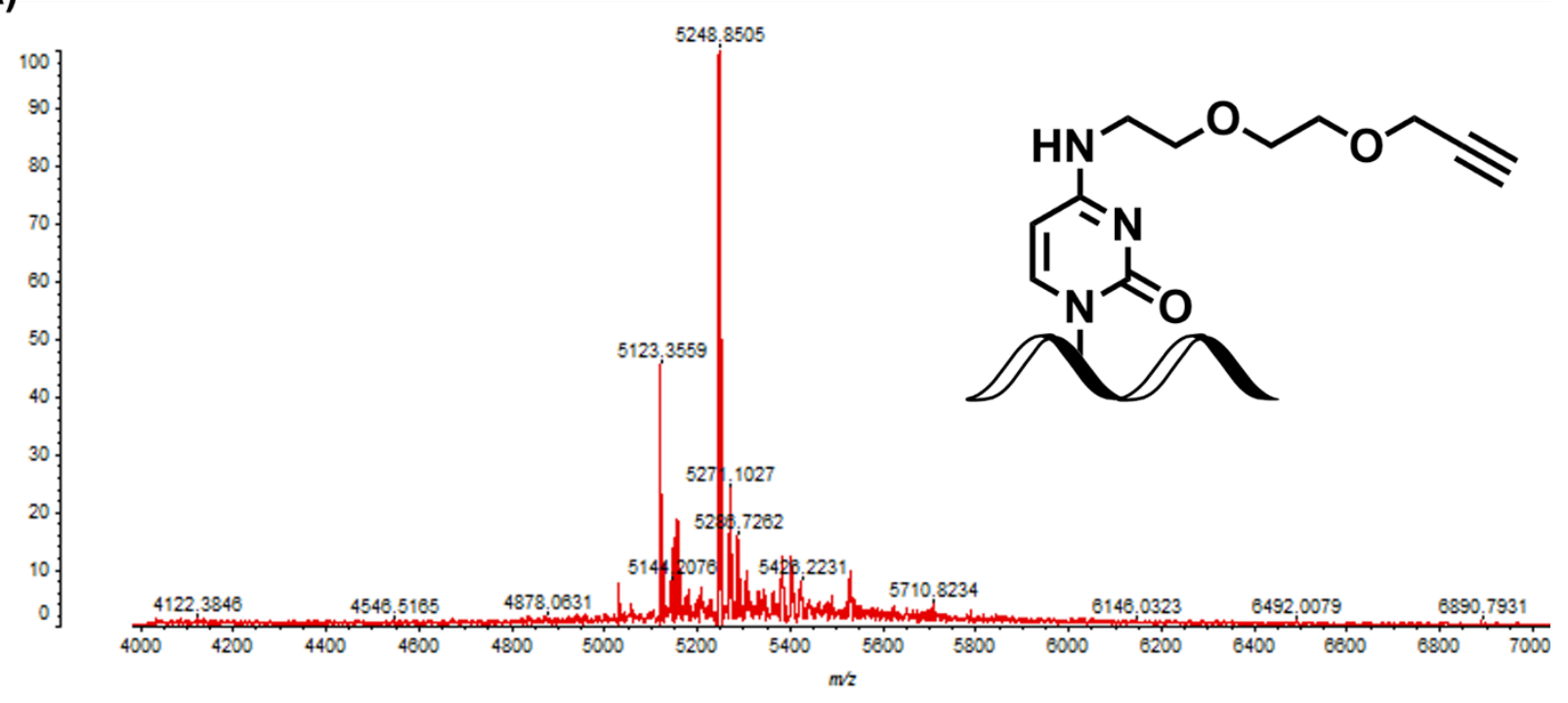

(b)

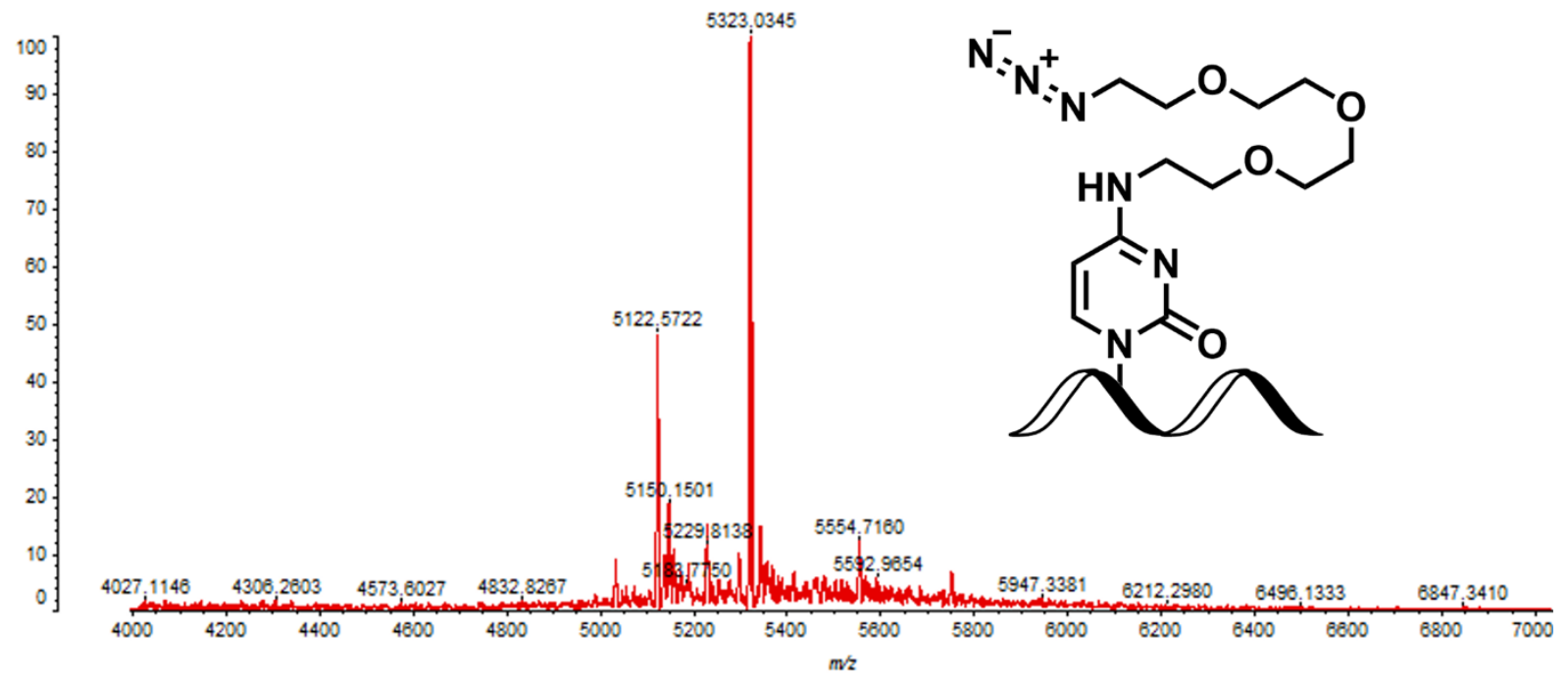

(c)

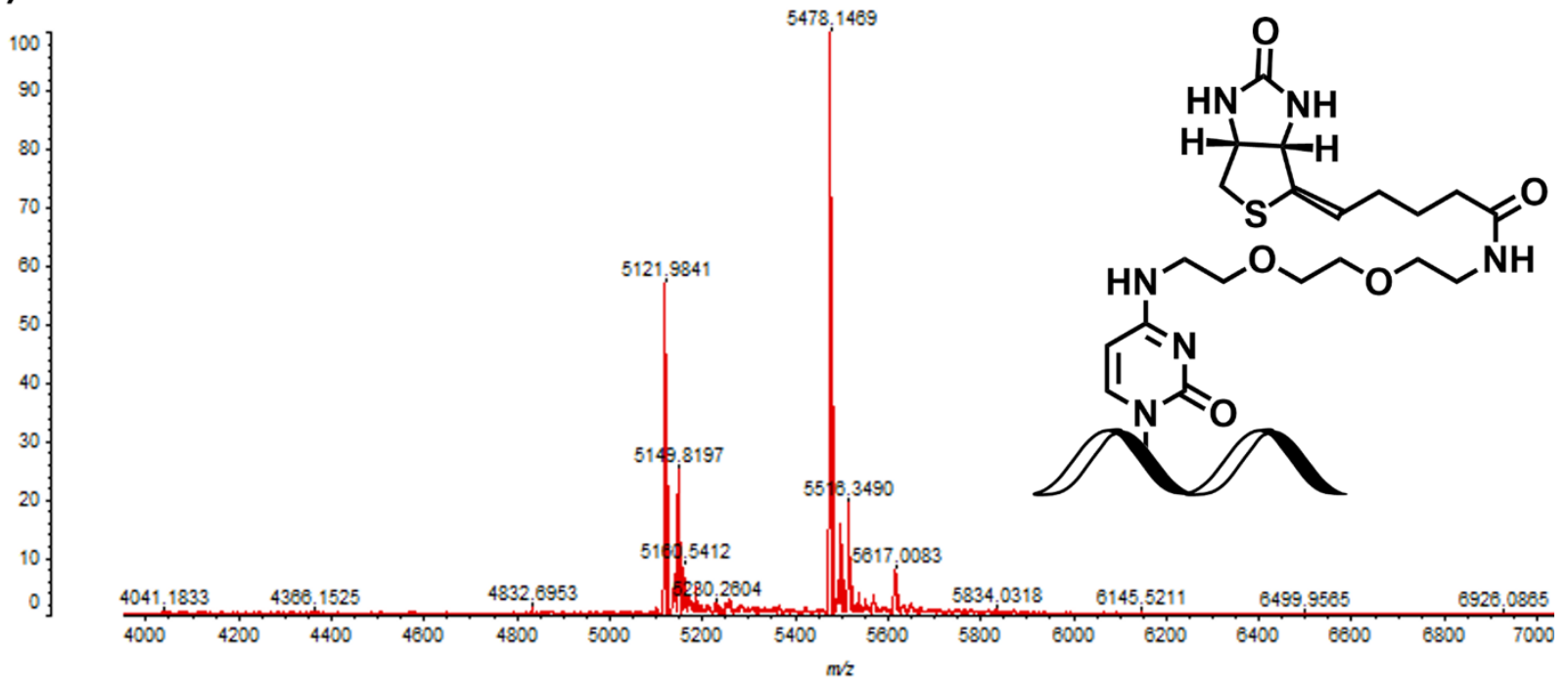


(d)

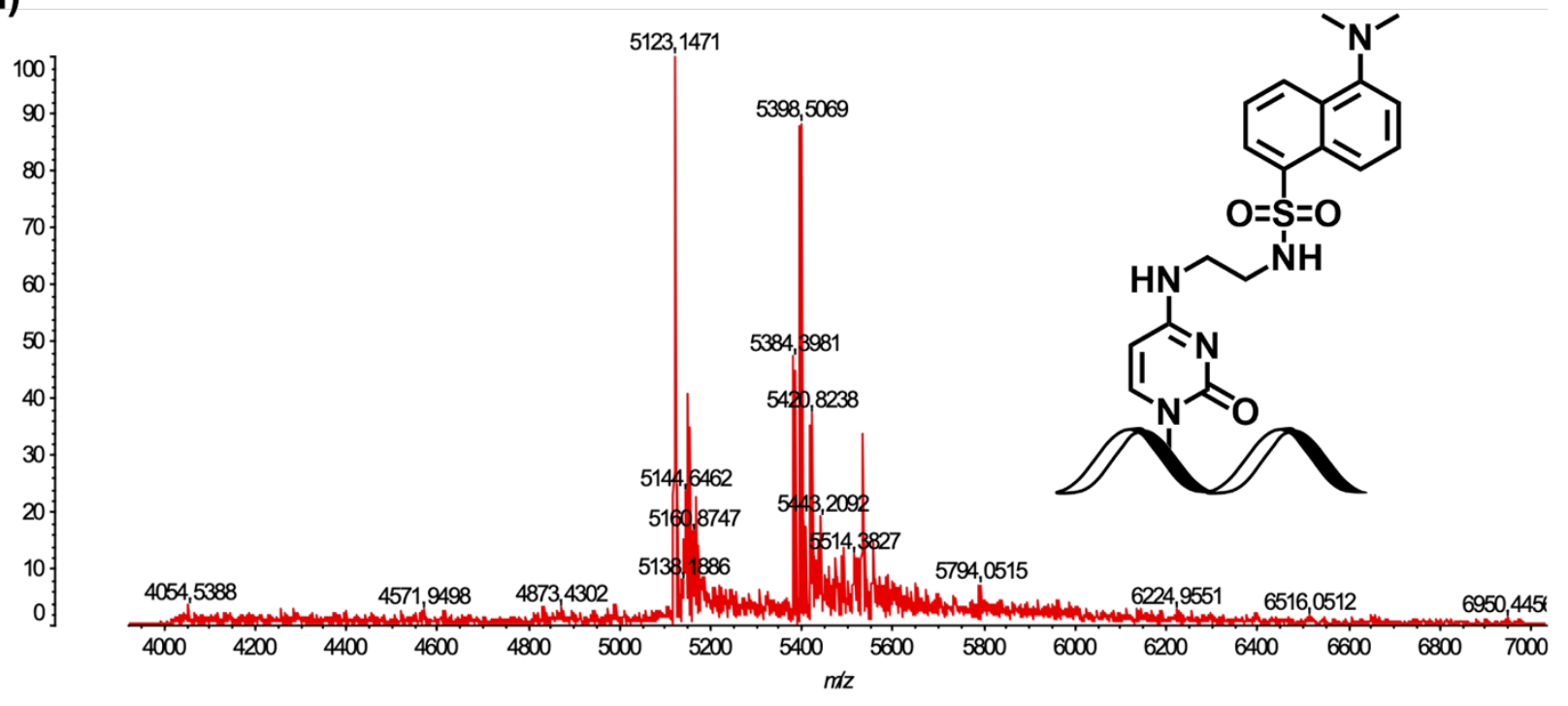

Figure S7. MALDI-TOF MS analysis of 4SdU ON derived with Alk-PEG2-NH2, Az-PEG3-NH2, Bio-PEG2$\mathrm{NH}_{2}$ and Dan-en, namely Alk-dC ON (a, calc. 5248.6, found 5248.8), Az-dC ON (b, calc. 5323.7, found 5323.0), Bio-dC ON (c, calc. 5479.6, found 5478.1) and Dan-dC ON (d, calc. 5398.5, found 5398.5).

(a)
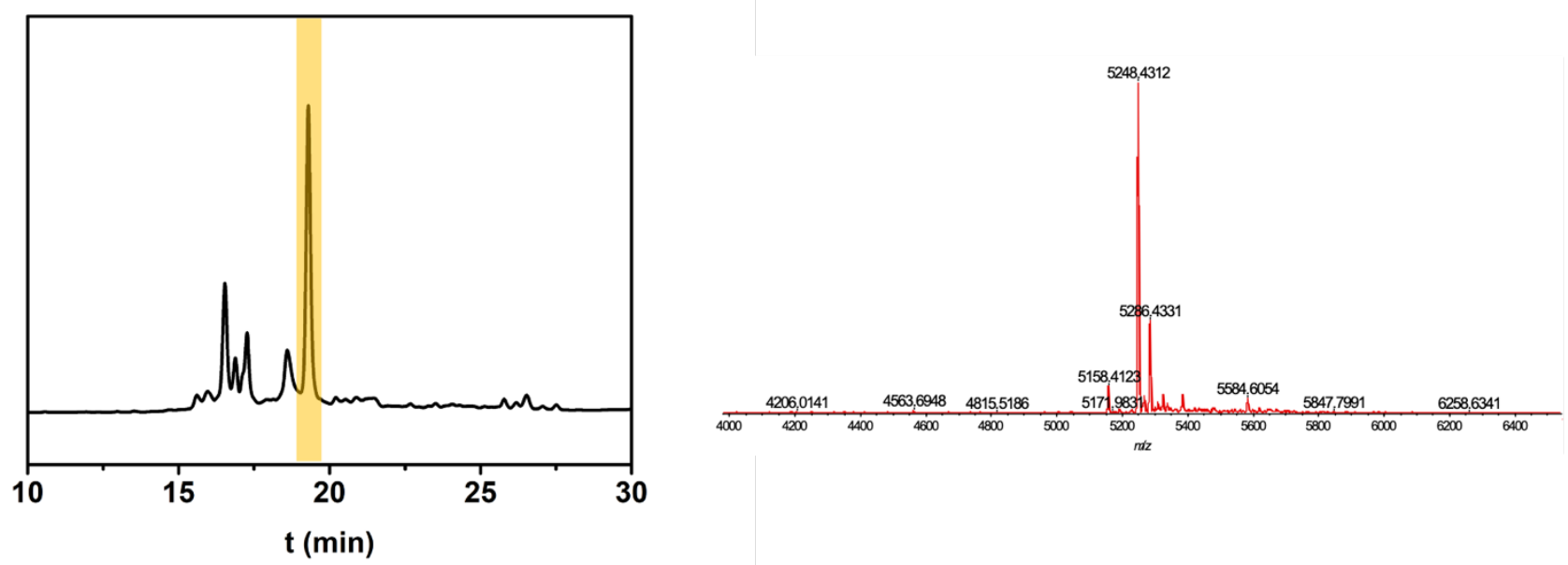

(b)
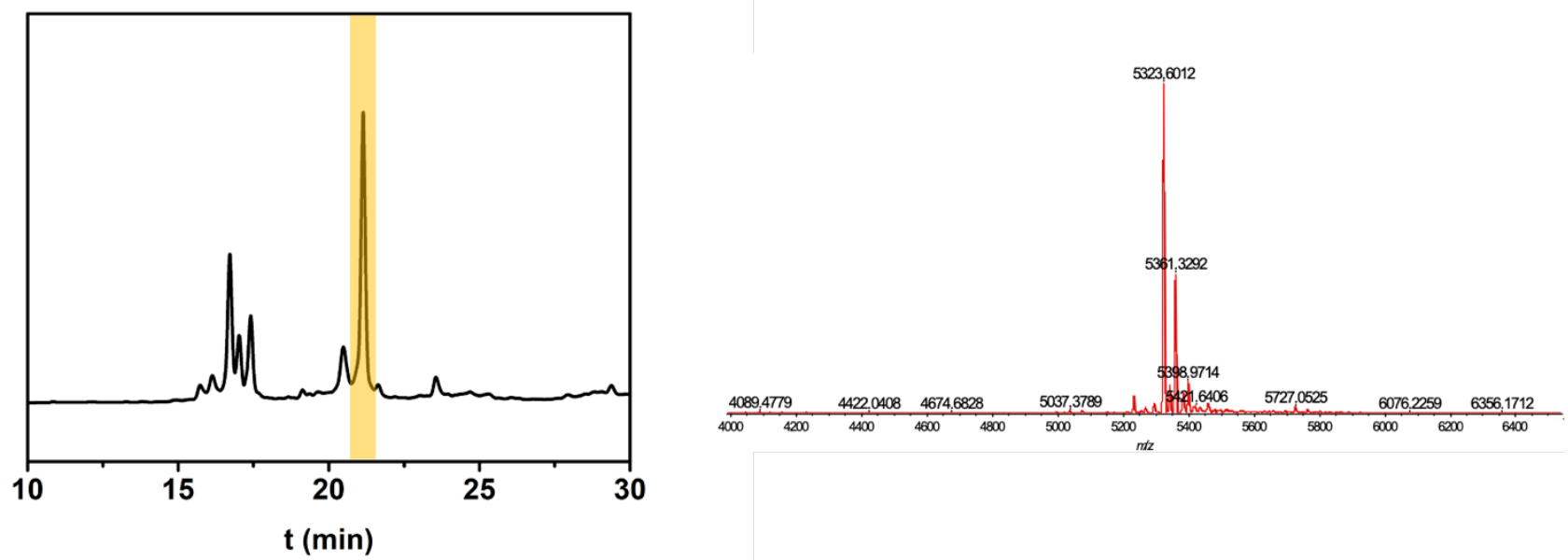
(c)
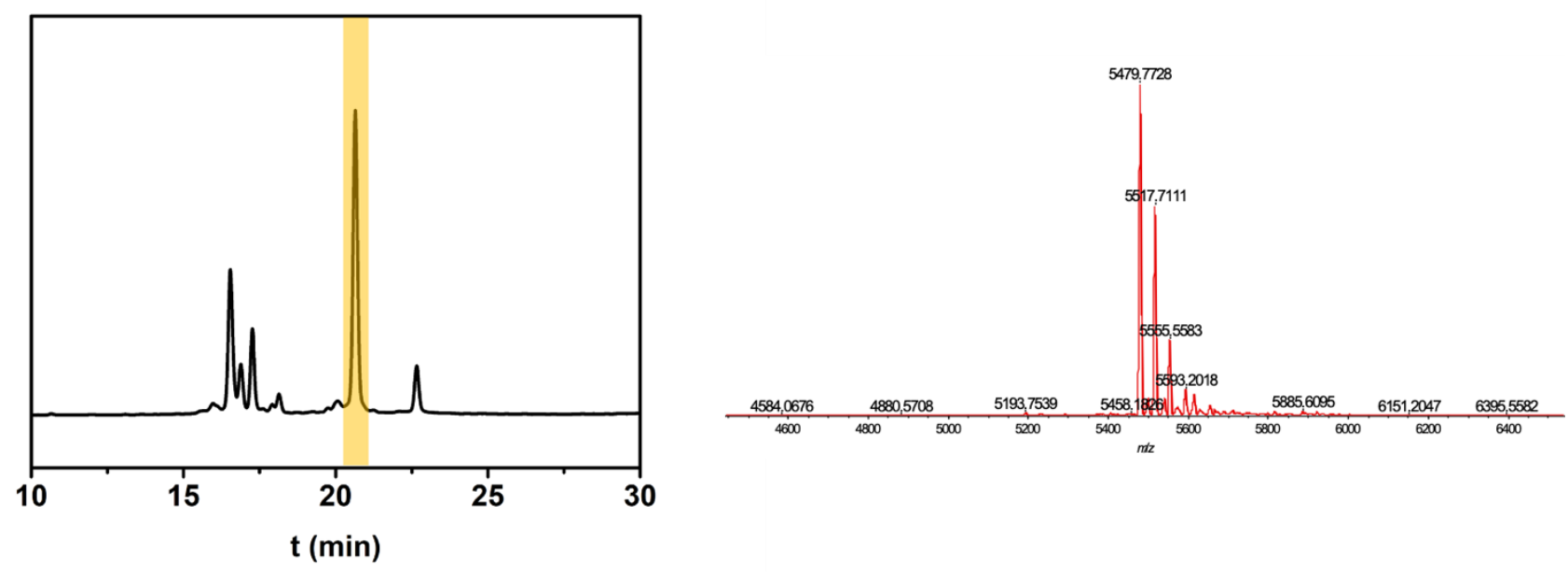

(d)
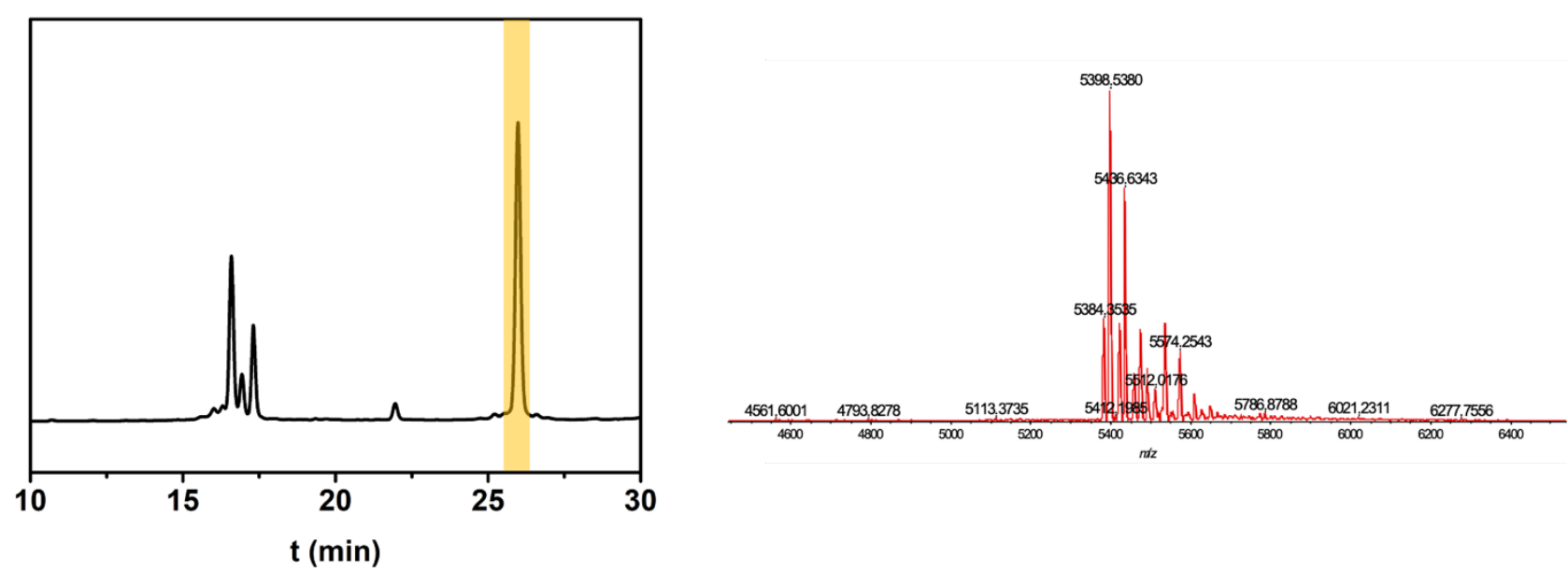

Figure S8. HPLC-UV analysis of Alk-dC ON (a), Az-dC ON (b), Bio-dC ON (c) and Dan-dC ON (d). The highlight peaks were collected for MALDI-TOF MS analysis. The pure products Alk-dC ON (calc. 5248.6, found 5248.4), Az-dC ON (calc. 5323.7, found 5323.6), Bio-dC ON (calc. 5479.6, found 5479.8) and Dan-dC ON (calc. 5398.5, found 5398.5) were isolated by HPLC. Additional peaks in mass spectra ([M] + $38 \mathrm{Da})$ were attributed to oligonucleotides replacing the hydrogen with potassium. 
(a)

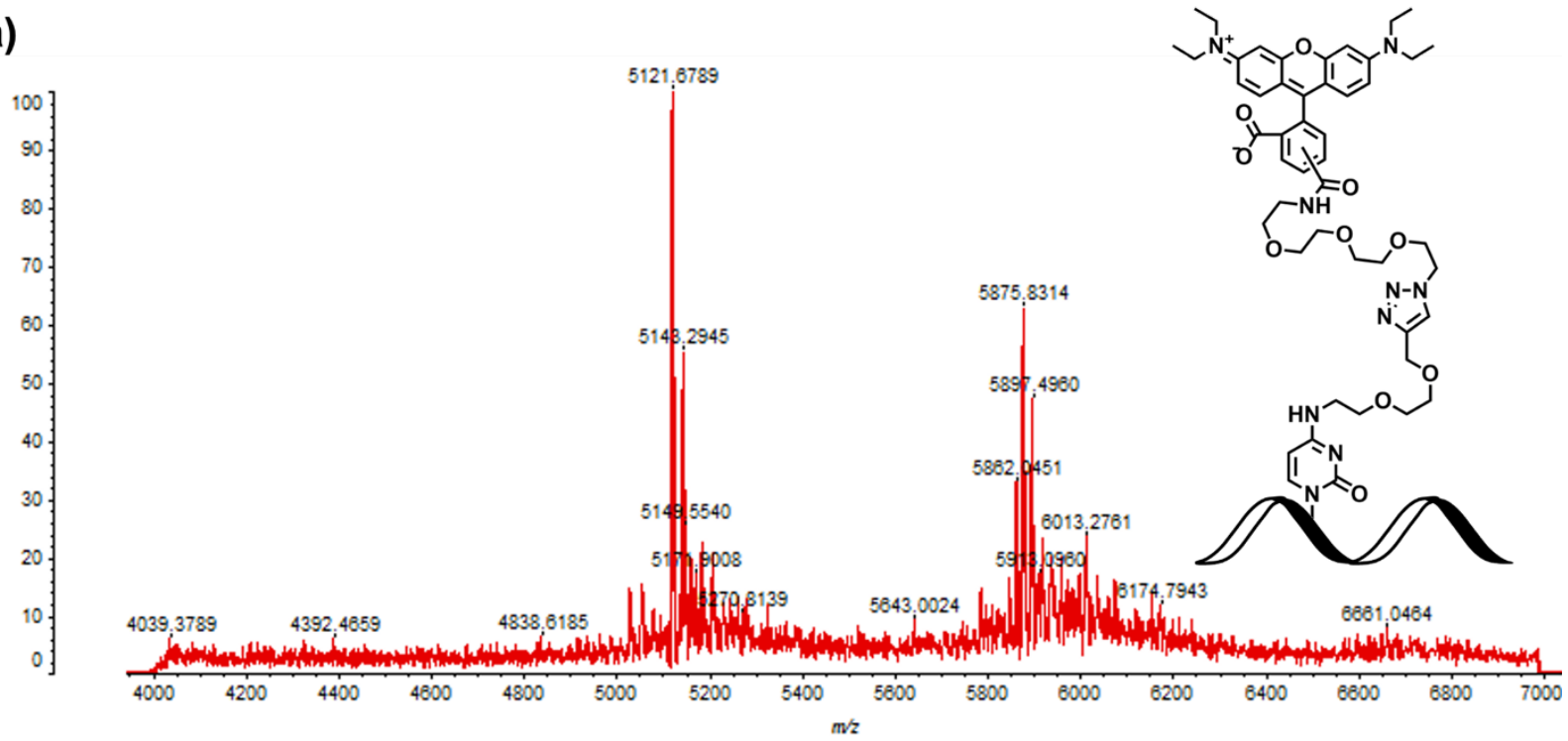

(b)

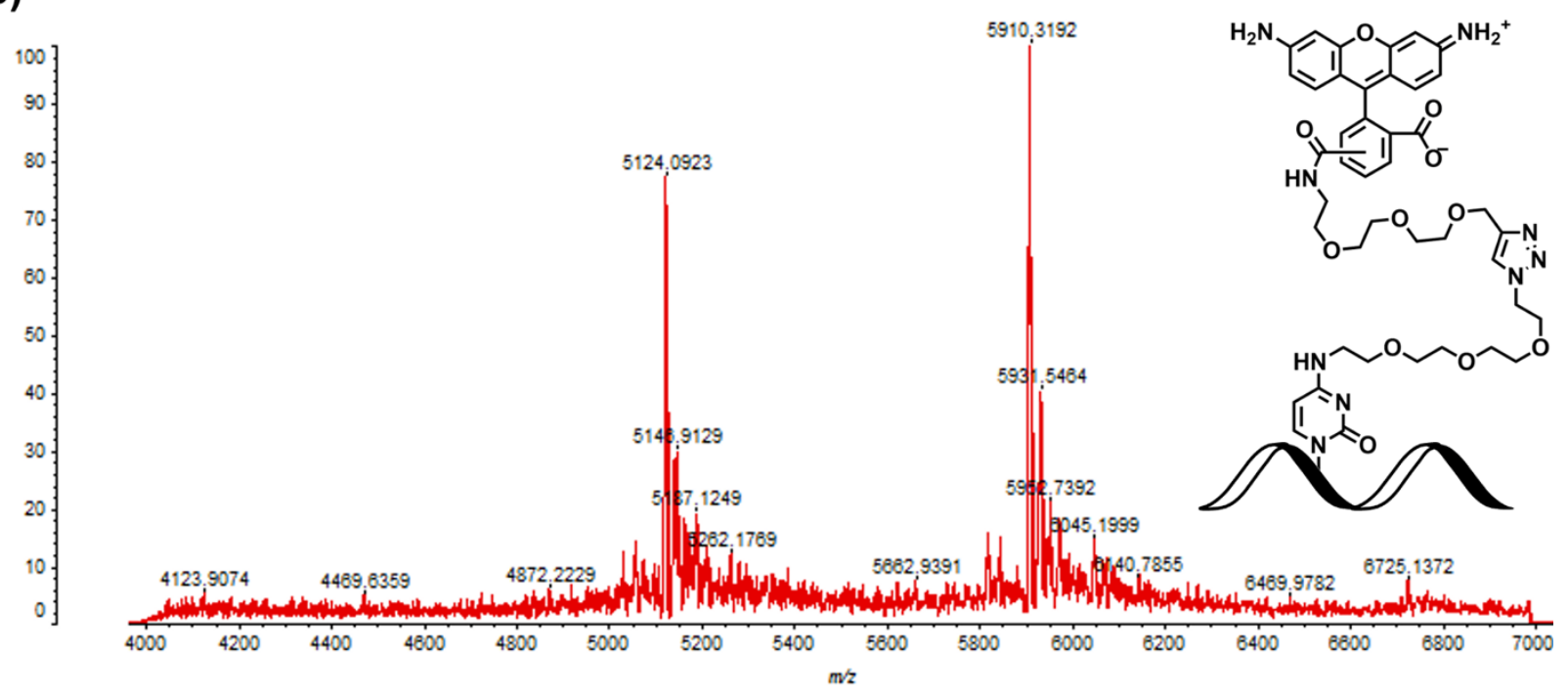

Figure S9. MALDI-TOF MS analysis of modified ONs clicked with alkyne or azide fluorophores, namely Az545-Alk-dC ON (a, calc. 5880.3, found 5875.8) and Alk488-Az-dC ON (b, calc. 5911.3, found 5910.3). 
(a)

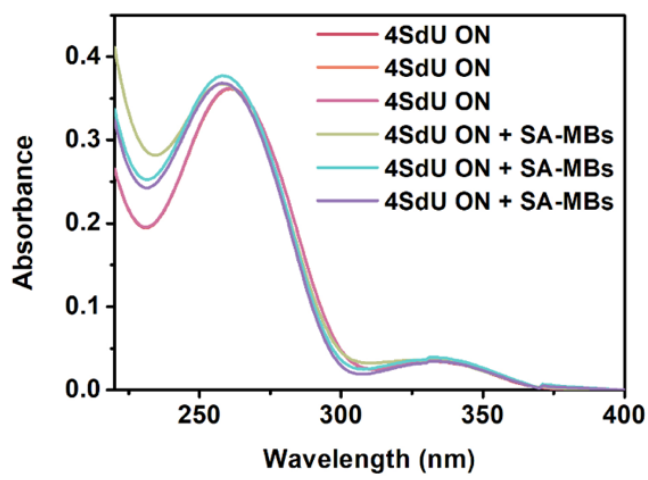

(b)

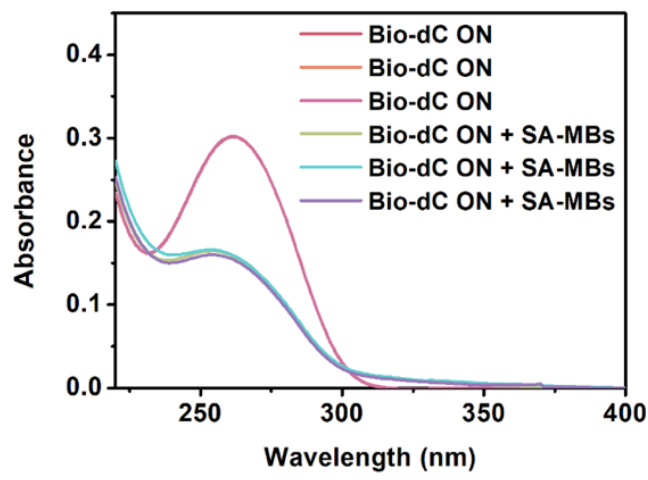

Figure S10. Absorption spectra of 4SdU ON (a) and Bio-dC ON (b) before and after treated with streptavidin magnetic beads adsorption.

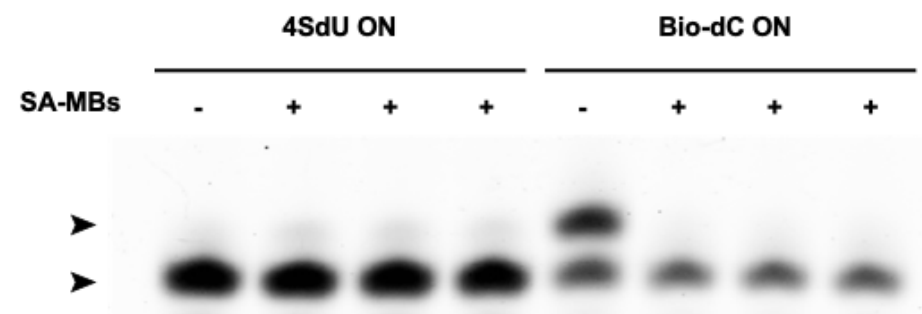

Figure S11. PAGE analysis of 4SdU ON and Bio-dC ON before and after treated with streptavidin magnetic beads adsorption.

\begin{tabular}{|c|c|c|c|c|c|c|c|}
\hline \multirow[b]{2}{*}{ Fluorophore } & \multicolumn{3}{|c|}{ dU ON } & \multicolumn{4}{|c|}{ 4SdU ON } \\
\hline & Dan-NH ${ }_{2}$ & $\mathrm{Fl}-\mathrm{NH}_{2}$ & Pyr-NH ${ }_{2}$ & 1 & Dan-NH ${ }_{2}$ & $\mathrm{FI}-\mathrm{NH}_{2}$ & Pyr-NH ${ }_{2}$ \\
\hline
\end{tabular}

Figure S12. PAGE analysis of dU ON and 4SdU ON treated with $\mathrm{NaIO}_{4}$ and amino-containing fluorophores. 


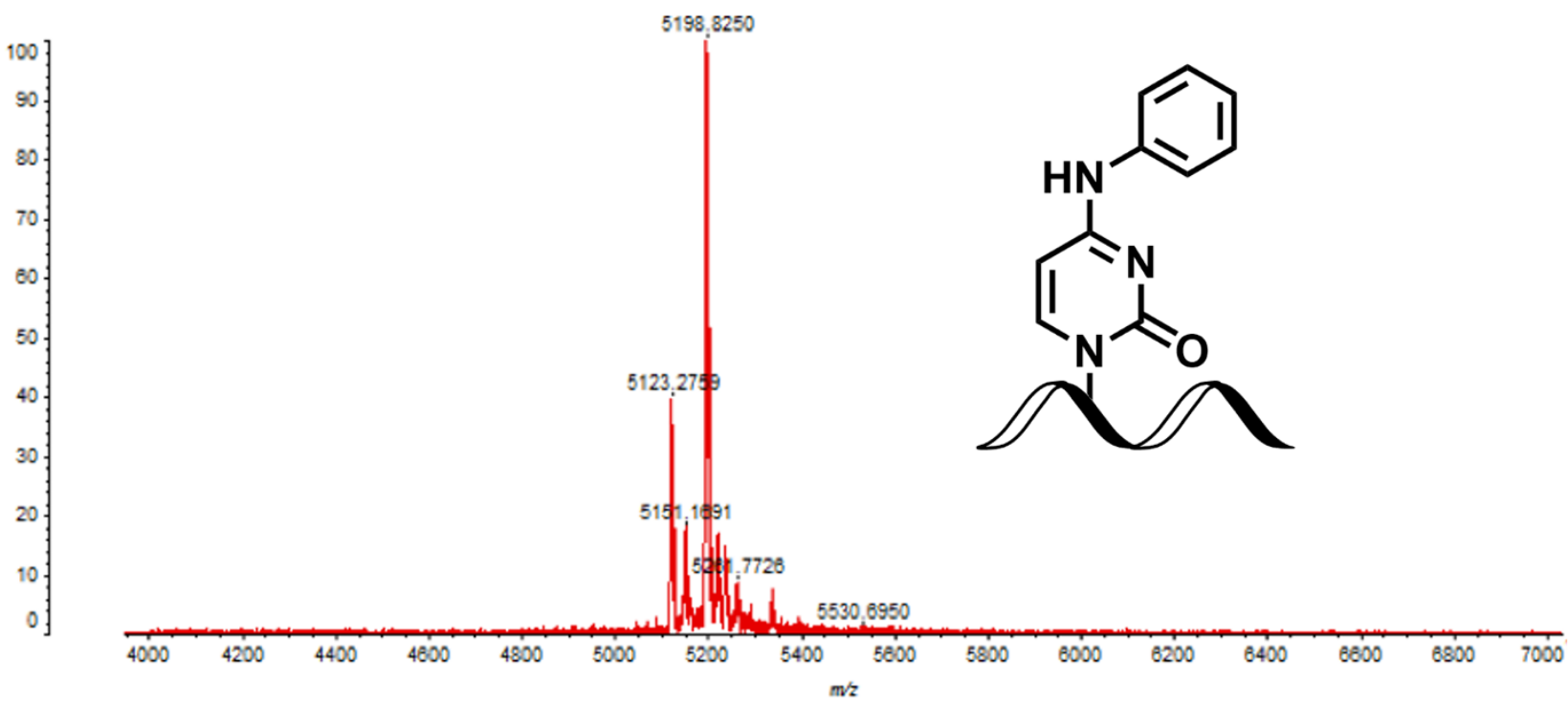

Figure S13. MALDI-TOF MS analysis of 4SdU ON derived with aniline ( $\left.\mathrm{Ph}-\mathrm{NH}_{2}\right)$, namely Ph-dC ON (a, calc. 5198.6, found 5198.8).
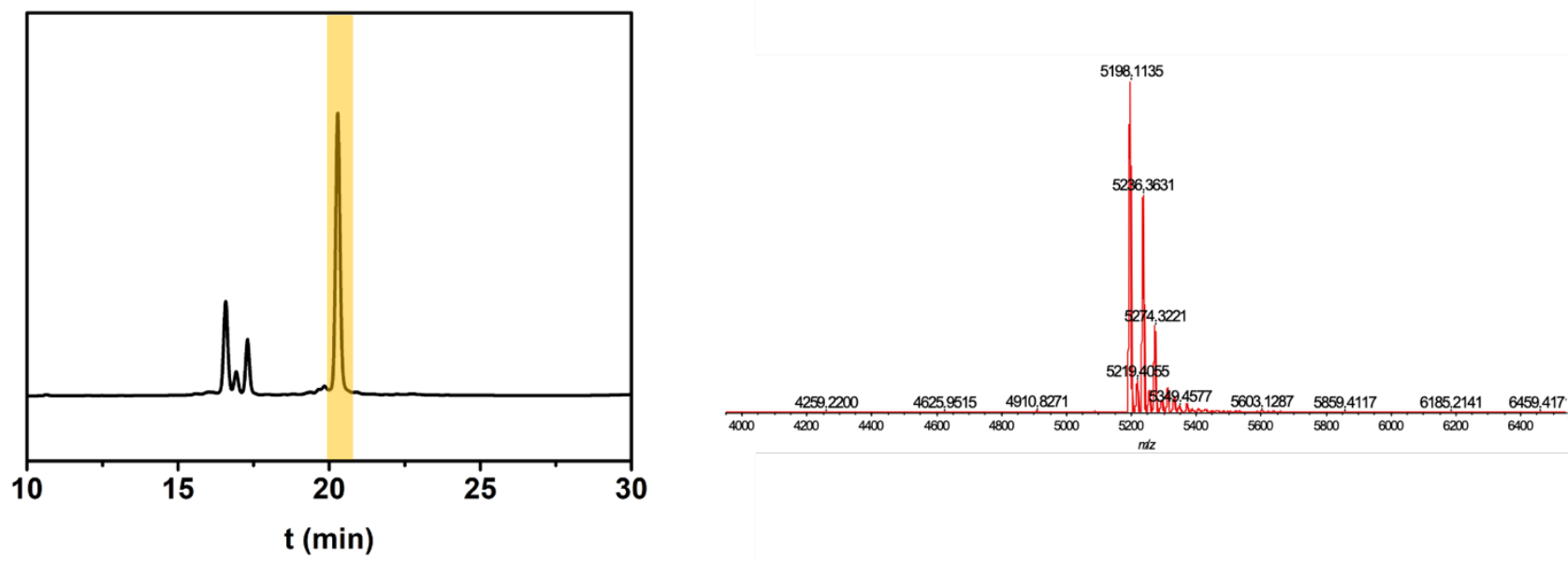

Figure S14. HPLC-UV analysis of Ph-dC ON. The highlight peak was collected for MALDI-TOF MS analysis. The pure product Ph-dC ON (a, calc. 5198.6, found 5198.1) was isolated by HPLC. Additional peaks in mass spectra ([M] + $38 \mathrm{Da}$ ) were attributed to oligonucleotides replacing the hydrogen with potassium. 
(a)

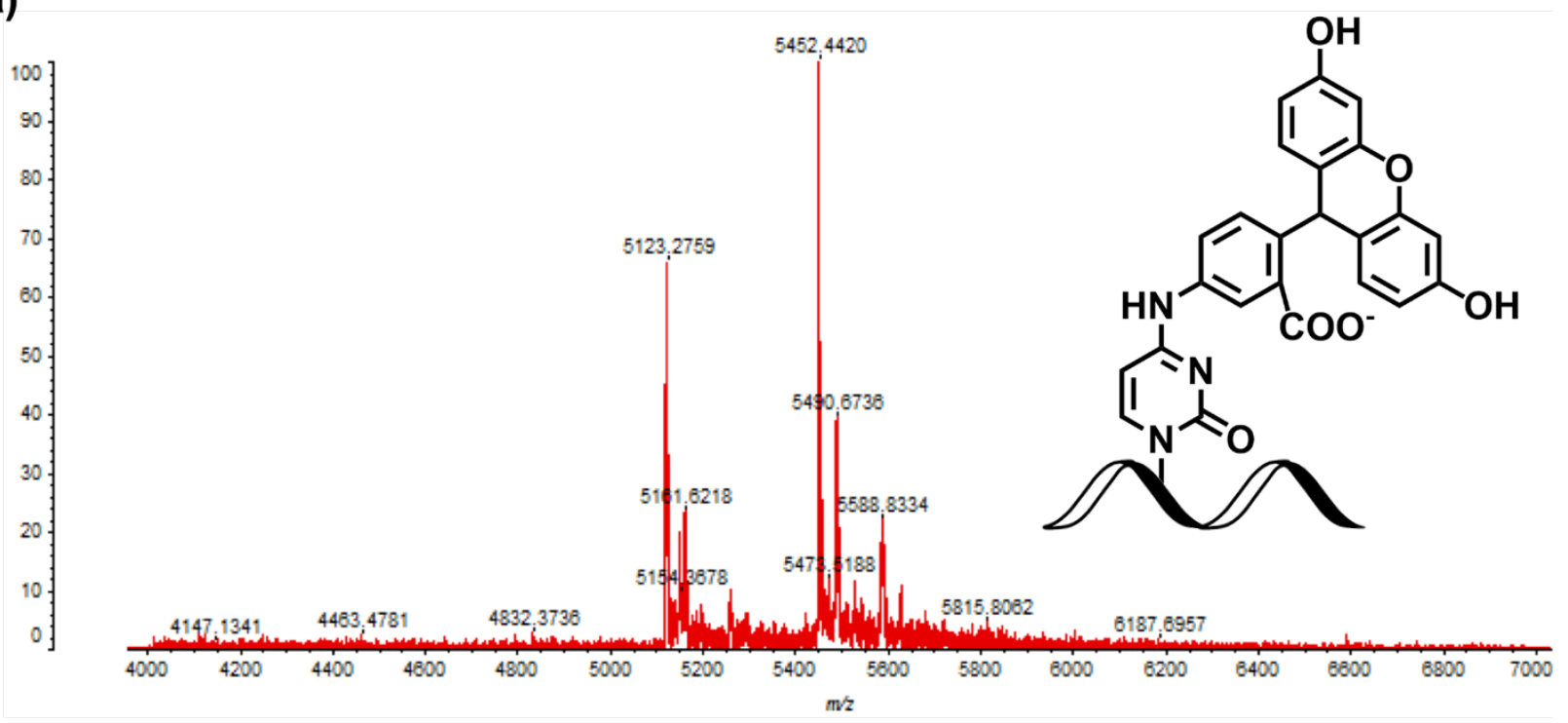

(b)

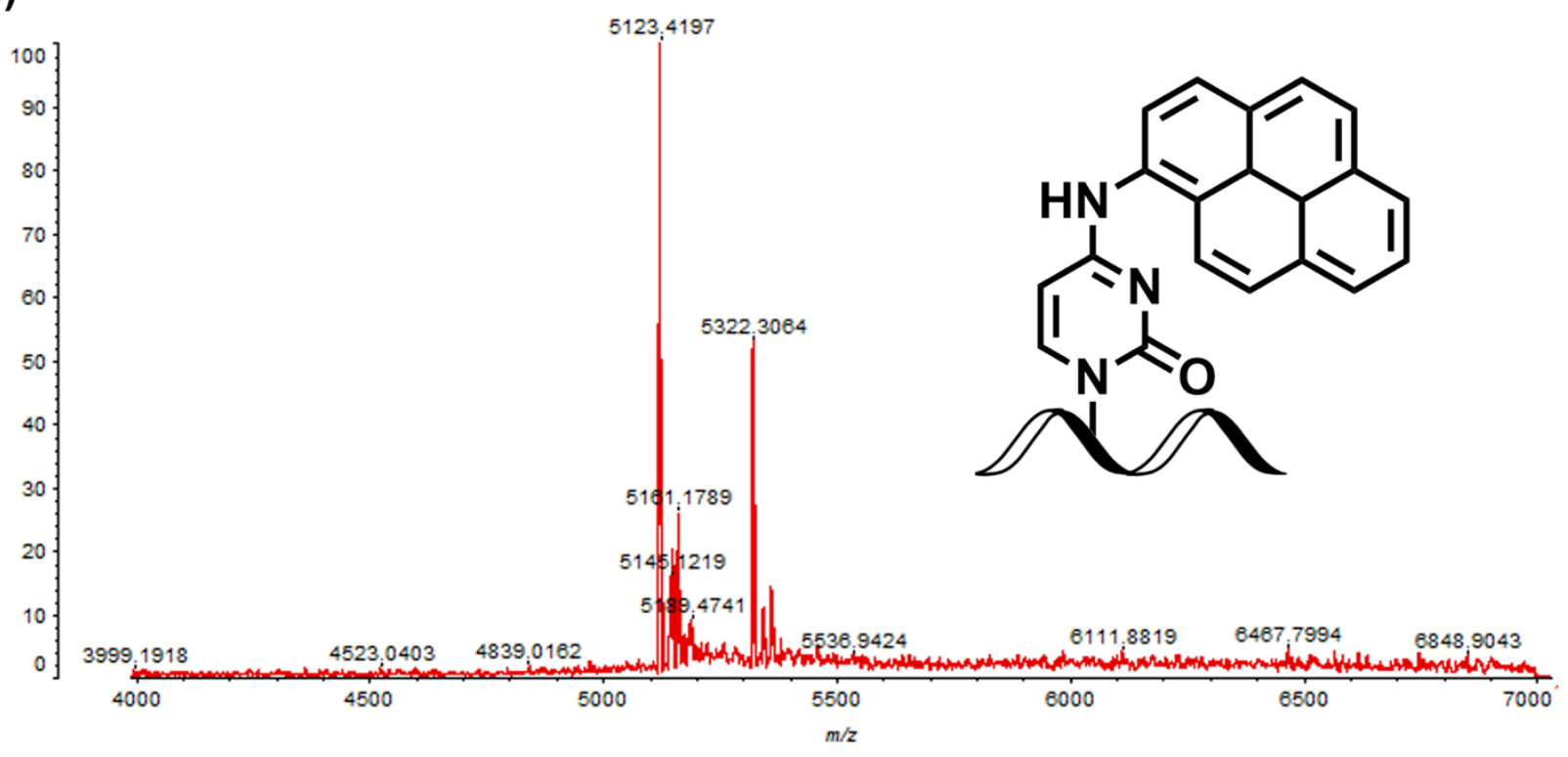

Figure S15. MALDI-TOF MS analysis of 4SdU ON derived with aminofluorescein $\left(\mathrm{Fl}-\mathrm{NH}_{2}\right)$ and aminopyrene (Pyr- $\mathrm{NH}_{2}$ ), namely Fl-dC ON (a, calc. 5452.7, found 5452.4) and Pyr-dC ON (b, calc. 5322.7, found 5322.3). 
(a)
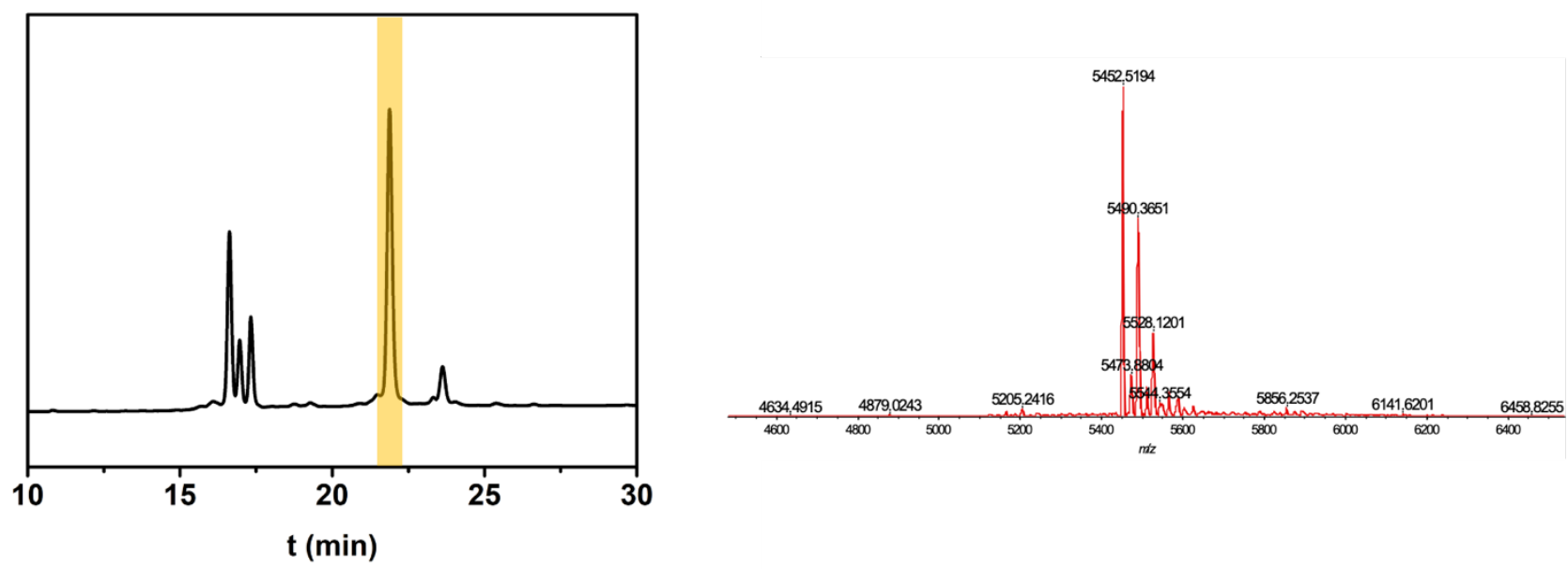

(b)
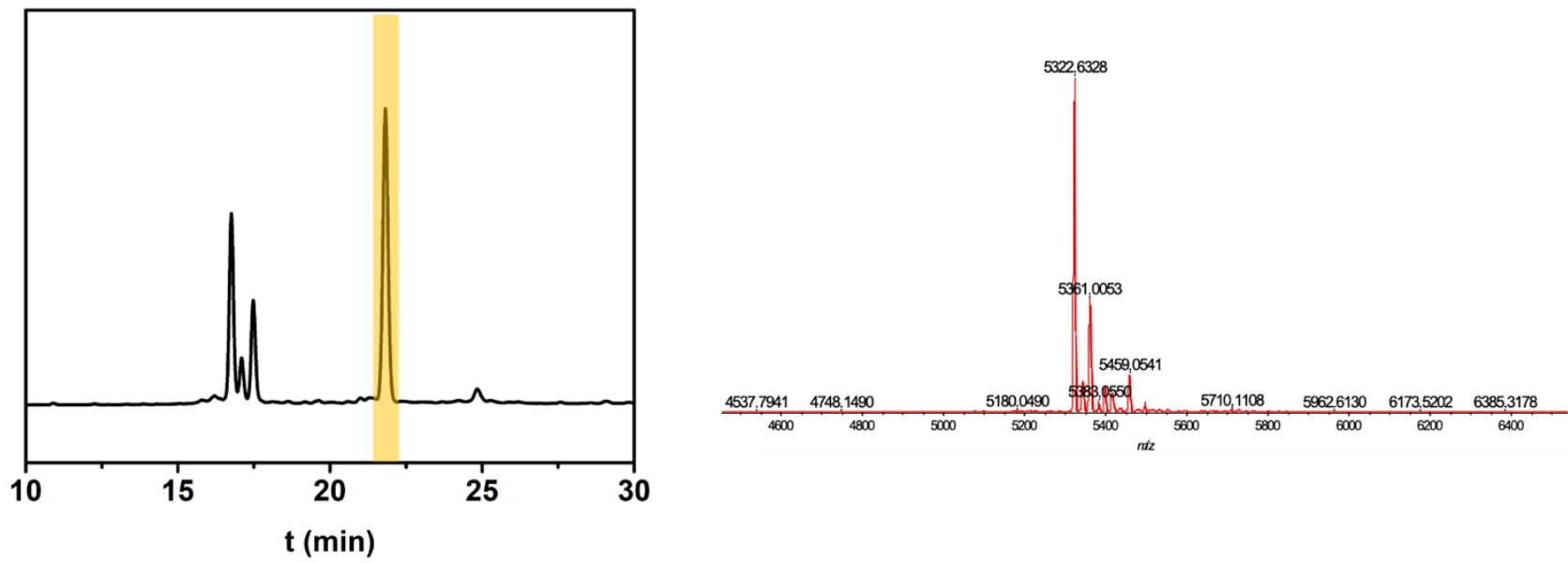

Figure S16. HPLC-UV analysis of Fl-dC ON (a) and Pyr-dC ON (b, purified by n-butanol extraction). The highlight peaks were collected for MALDI-TOF MS analysis. The pure products Fl-dC ON (calc. 5452.7, found 5452.5) and Pyr-dC ON (calc. 5322.7, found 5322.6) were isolated by HPLC. Additional peaks in mass spectra ([M] + $38 \mathrm{Da})$ were attributed to oligonucleotides replacing the hydrogen with potassium. 